
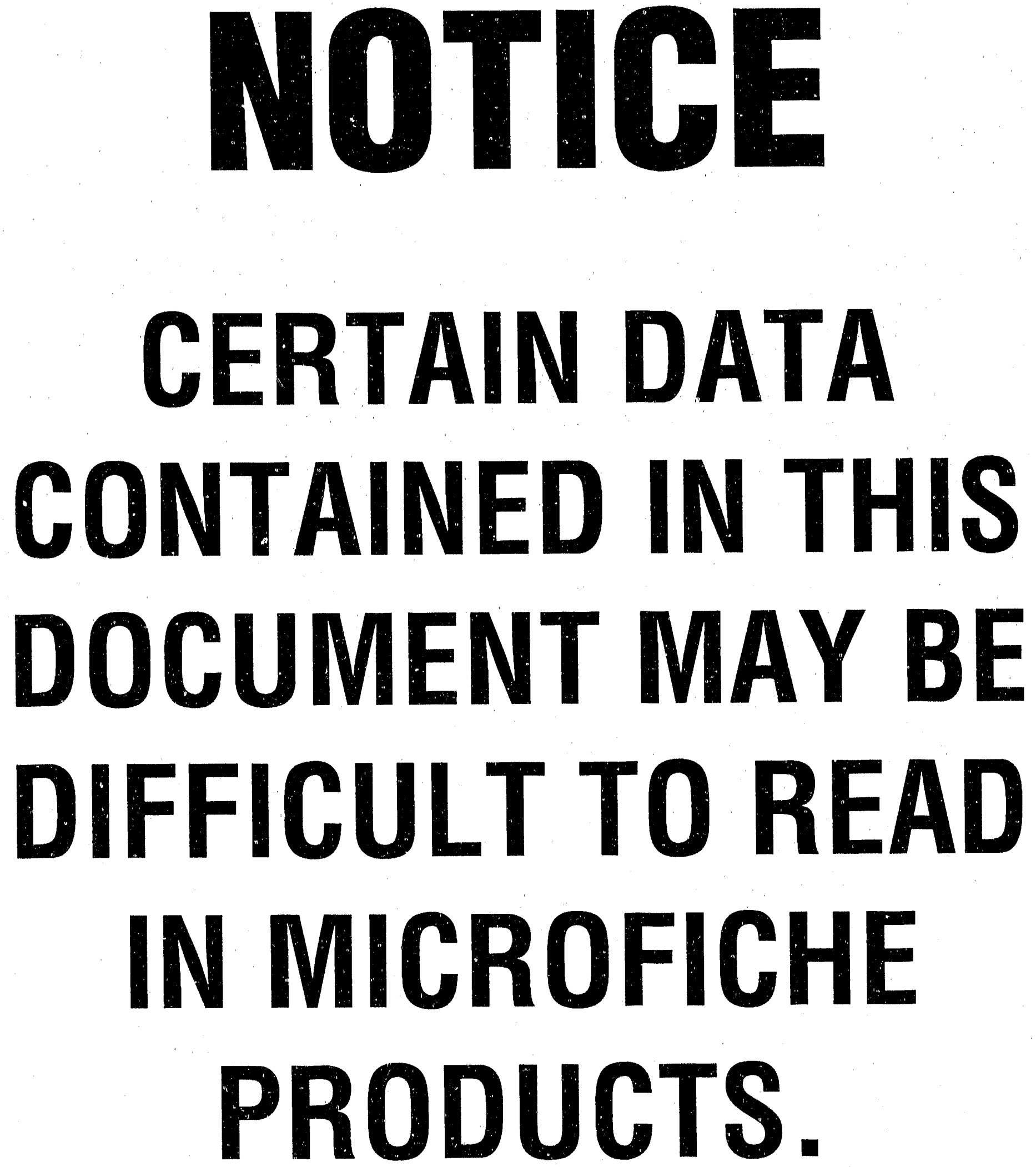


\section{LA-UR $90-3253$}

AUTHOR(S):

D. S. Sivia

W. A. Hamilton

G. S. Smith

DE9 1000215
SUBMITTED TO:

Proceedings:

"Maximum Entropy and Bayesian Methods: Laramie 1990"

T. Grandy \& L. Schick eds., Kluwer Academic Publishers

\section{DISCLAIMER}

This report was prepared as an account of work sponsored by an agency of the United States Government. Neither the United States Government nor any azency thereof, nor any of their employees, makes any warranty, express or implied, or assumes any legal liability or responsibility for the accuracy, completeness, or usefulness of any information, apparatus, product, or process disclosed, or represents that its use would not infringe privately owned rights. Reference herein to any specific commercial product, process. or serviç; by trade name, trademark, manufacturer, or otherwise does not necessarily constıtute or imply its endorsement, recommendation, or favoring by the United States Government or any agency thereof. The views and opinions of authors expressed herein do not necessarily state or reflect those of the United States Government or any agency thereof.

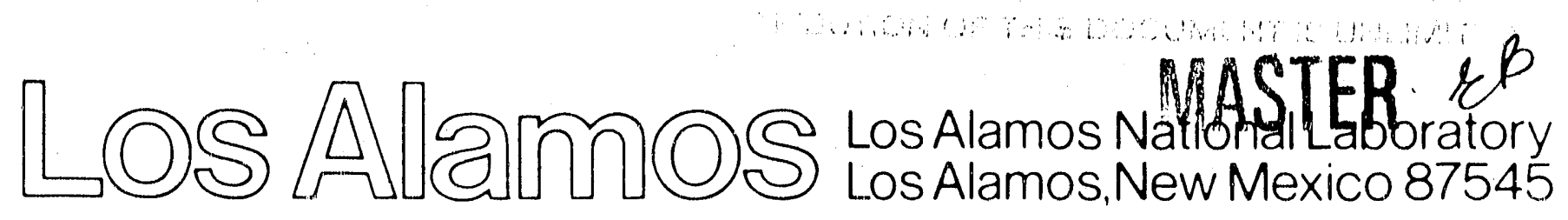




\title{
BAYESIAN SPECTRAL ANALYSIS OF REFLEC'TIVITY DATA
}

\author{
D. S. Sivia, W. A. Hamilton and G. S. Smith \\ Theoretical Division and Manuel Lujan Jr Neutron Scattering Center \\ Los Alamos National Laboratory \\ Los Alamos, New Mexico 87545, US.A
}

\begin{abstract}
The analysis of neutron and $x$-ray reflectivity data to obtain scattering-length density profiles is akin to the notorious phaseless Fourier problem, well-known in many fields such as crsytallography. Current methods of analysis culminate in the refinement of a few parameters of a functional model, and are often preceded by a long and laborious process of trial-and-error, A discussion of the use of maximum entropy for obtaining "free-form" solutions of the density profile, as an alternative to the trial-and-error phase when a functional model is not available, and the suggestion for a novel experimental procedure designed to alleviate the ambiguity problems inherent in traditional reflectivity measurements, are given in Sivia et al. (1990). In this paper we consider the Bayesian spectral analysis approach, which is appropriate for optimising the parameters of a simple (but adequate) type of model when the number of parameters is not known.
\end{abstract}

\section{Introduction}

A wide variety of interfaces of scientific and technological interest exhibit depth profile structure on length scales of tens to thousands of Angstrom. Examples include: model biological lipid membranes, analogous to the walls of living cells; semi-conductor multilayers of importance to the electronics industry; organic thin-film chemical sensors; conjugated polymers for electro-optic devices; and thin-film superconductors. This size range is an order of magnitude less than is accessible by conventional optical techniques. One method of probing interfacial structures on these length scales is to measure the interforence effects of the depth profile on the reflection of short wavelength radiation. This extension of visible range optical techniques to shorter wavelengths has been vigorously pursued over the last few years in $x$-ray reflectometry, and also by the increasingly popular complementary technique of neutron reflectometry.

In Section 2 we consider reflectivity from a data analysis point-of-view and in Section 3 we present the Bayesian spectral analysis approach to the problem. This is a generalised form of model-fitting where the solutions are restricted to a broad class of models rather than to a particular model. The choice of the type of modela encodes considerably more prior knowledge about the nature of the depth profile than does free-form approach (such as MaxEnt), while still allowing considerably more flexibility than conventional model refinement. We conclude with Section 4. 


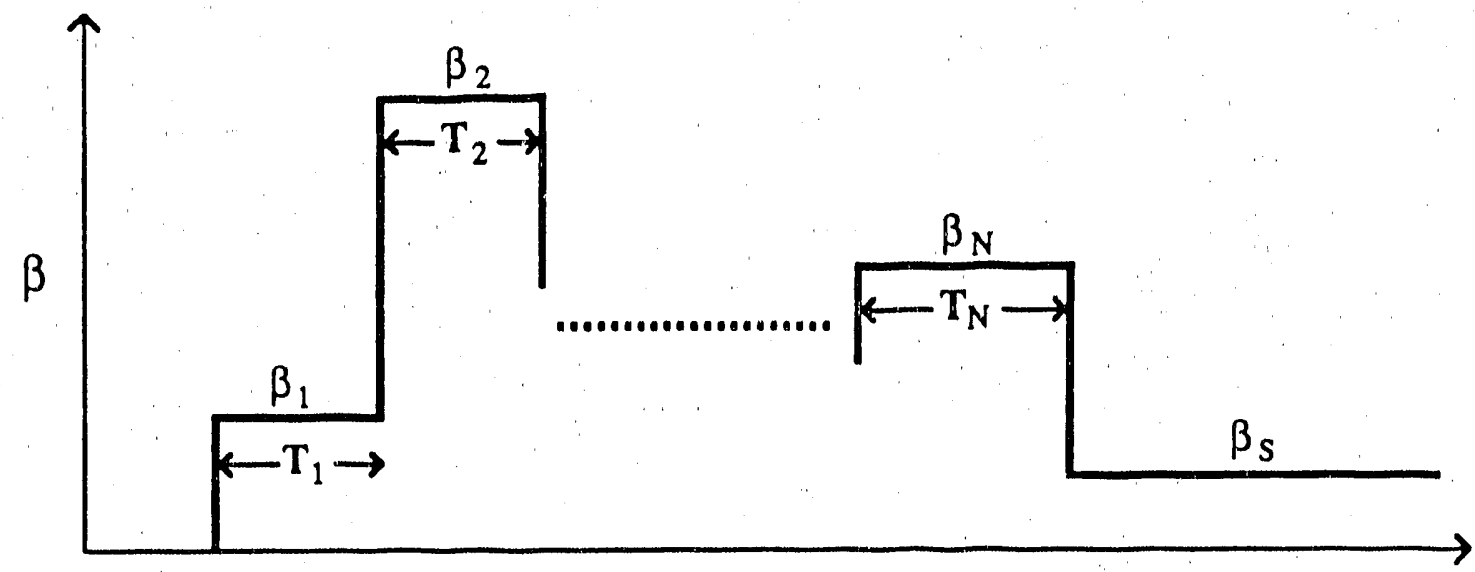

Figure 2. A simple, but (hopefully) adequate, representation of the scattering-length density profile based on the prior knowledge that layered structures have histogram-like depth profiles.

profile by constructing a hypothesis space defined by the "heights and widths" of a few layers as illustrated in Fig. 2. We could make a more elaborate model for the density profile by assigning a "roughness" parameter to each interface, and a "tilt angle" to each layer, but we ignore such complications for simplicity in this paper (the generalisation being straight-forward). This is also in keeping with the spirit of Bayesian spectral analysis where we are trying to estimate the parameters of a simple, but adequate, type of model when the number of parameters is not known. By using our additional prior knowledge to construct a much smaller hypothesis space (than that used in the free-form approach) we hope to obtain more accurate estimates of the depth profile, the reliability of which is also easier to quantify.

For this formulation of the problem, our best estimate of the density profile is obtained by maximising the posterior p.d.f.:

$$
\operatorname{prob}\left[T_{1}, T_{2}, \ldots, T_{N}, \beta_{1}, \beta_{2}, \ldots, \beta_{N}\left|R(Q), \beta_{S}\right|\right.
$$

where $\left\{T_{i}, \beta_{i}\right\}$ are the set of heights and widths of the $N$ layers describing the density profile, $\beta_{S}$ is the acattering-length density of the substrate (assumed known) and $R(Q)$ are the reflectivity data. If the number of layers was known, this would be a straight-forward problem of parameter eatimation (in principle). However, wo do not know NI So, we need to compute prob $\left[N \mid R(Q), \theta_{s}\right]$.

The mathematics presented below, to compute the posterior probability for the number of layers, is essentially the same as that found in Chapter 5 of Jeffreys (1939). It involves the use of Bayes' theorem and marginalisation, and requires a number of assumptions and approximations to be made in order to obtain an analytic solution. Although the assumptions and approximations are always open to question, they are not unusual in that they are always implicit when using the commonly-used least-squares analysis.

We begin by using Bayes' theorem: 

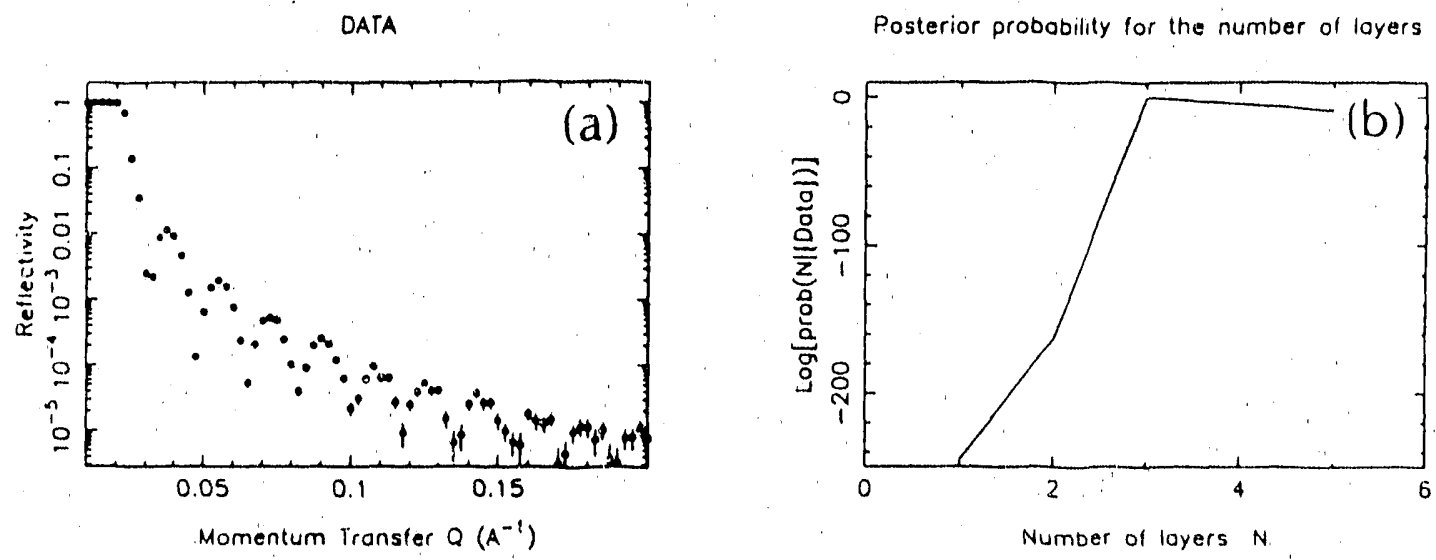

Posterior probability for the number of loyers

Best-fit density prolile assuming no. of layers $=3$
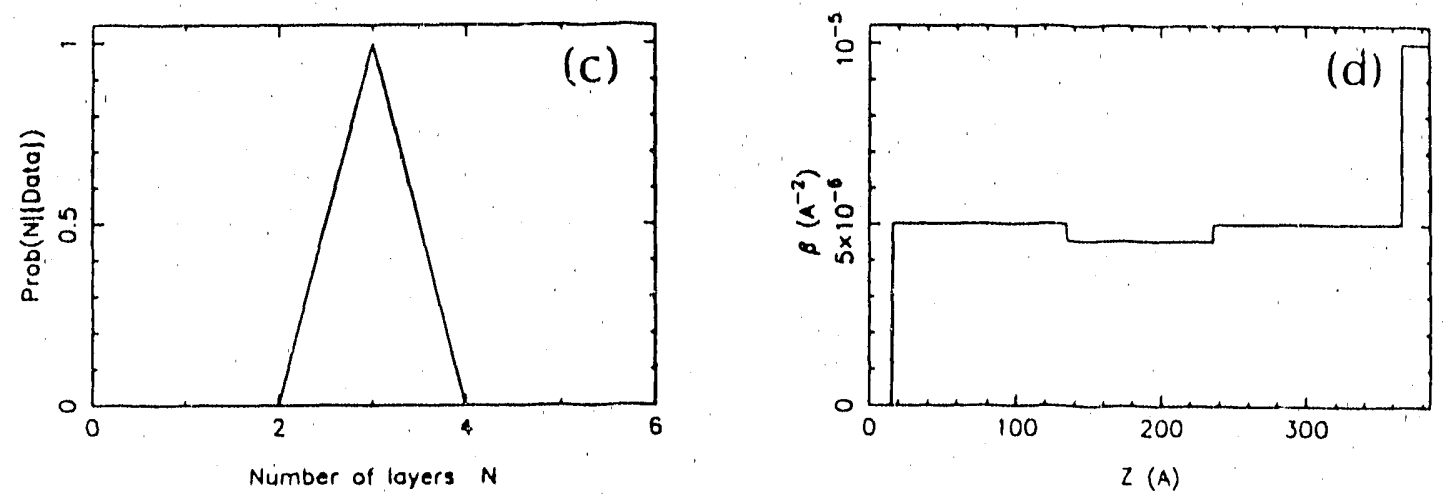

Figure 3. (a) Simulated reflectivity data. (b) \& (c) The posterior probability for the number of layers plotted on a logarithmic and linear scale respectively. (d) Best estimate of the density profile.

$$
\operatorname{prob}[N \mid R(Q)]=\frac{\text { Constant. } N !}{\left[\left(\beta_{\max }-\beta_{\min }\right) \cdot T_{\max }\right]^{N}} \times \frac{e^{-x_{\min }^{2} / 2}}{\sqrt{\operatorname{Det}\left(\nabla \nabla \chi^{3}\right)}}
$$

where $\operatorname{Det}\left(\nabla \nabla \chi^{2}\right)$ is the determinant of the Hessian matrix.

\section{AN EXAMPLE}

Fig. 3(a) shows simulated reflectivity data generated from a density profile consisting of a few layers; the simulated data suffer from typical instrumental resolution broadening and noise. Figs. 3 (b) and (c) show the posterior probability for the number of layers, computed according to the formula derived above. $T_{\max }$ was taken to be the actual width of the sample ( $350 \AA$ ) on the basis that, in general, it is very difficult to find the best-fit solutions if a good eatimate of the overall width is not available. $\beta_{\max }$ was set to $10^{-5}$ $A^{-2}$ in accordance with prior knowledge about the neutron scattering-lengths of atoms (the simulated density profile constructed to comply), $\beta_{\min }$ was set zero indicating prior 
the complex topology of the likelihood function for this non-linear problem means that it is very hard to find the best-fit solution, even when one knows the number of layers; a full parameter-space search entails cosmological tirne-scales even for models with only 4 or 5 layers! In practice we have found that our chances of success are greatly increased (above zero) when we have a good estimate of the total width of the sample.

\section{Conclusions}

Bayesian spectral analysis is a very powerful technique. It is a generalised form of modelfitting, which is appropriate for optimising the parameters of a simple (but adequate) type of model when the number of parameters is not known. In this paper we have the example of estimating the density profile for a layered sample, when the number of layers is unknown, but there are numerous other applications. Gull (1988), for example, addresses the problem of fitting a polynomial to a pertinent set of finite and noisy data, (again) when the order of the polynomial is not known.

This method of analysis encodes more prior knowledge about the object of interest than does a free-form approach, while still allowing a considerable amount of flexibility over traditional model refinement. Although we are often hesitant to commit ourselves to (even a clase of) simple functional models, the advantages of working in such a reduced hypotherio space are that we are able to obtain results which are more reliable and easier to quantify. It is in the nature of scientific inference that the best we can do is show that a theory (or choice of hypothesis space) is adequate (rather than "the truth"); if it is not adequate, probability theory will tell us so!

ACKNOWLEDGMENTS. We thank Roger Pynn for his encouragement and useful discussions. This work was supported by the Office of Basic Sciences of the U.S. Department of Energy.

\section{REFERENCES}

Felcher, G.P. (1988), 'Thin-Film Neutron Optical Devices', SPIE, 983.

Gull, S.F. (1988). 'Bayesian Inductive Inference and Maximum Entropy', in Maximum Entropy and Bayerian Methods in Science and Engineering (Vol. 1), eds. G.J. Erickson \& C.R. Smith, Kluwer Academic Publishers.

Jeffreys, H. (1939). Theory of Probability, Oxford University Press. Third edition: 1983.

Jaynes, E.T. (1986). 'Bayesian Methods - an Introductory Tutorial', in Maximum Entropy and Bayesian Methods in Applied Statistics, J.H. Justice ed., Cambridge University Press.

Lekner, J. (1987). Theory of the Reflection of Electromagnetic and Particle Waves, Martinez Nijof Publishers, Dordrecht, Holland.

Russell, T.P. (1990). 'X-ray and Neutron Reflectivity for the Investigation of Polymers', submitted to Materials Research Reports.

Sivia, D.S., Hamilton, W.A. and Smith, G.S. (1990). 'Analysis of Neutron Reflectivity Data: Maximum Entropy, Bayesian Spectral Analysis and Speckle Holograhy', Physica B, eds. G.P. Felcher and T.P. Russell (in press).

Thorburn, W.M. (1918). Mind, 27, 345-353. 

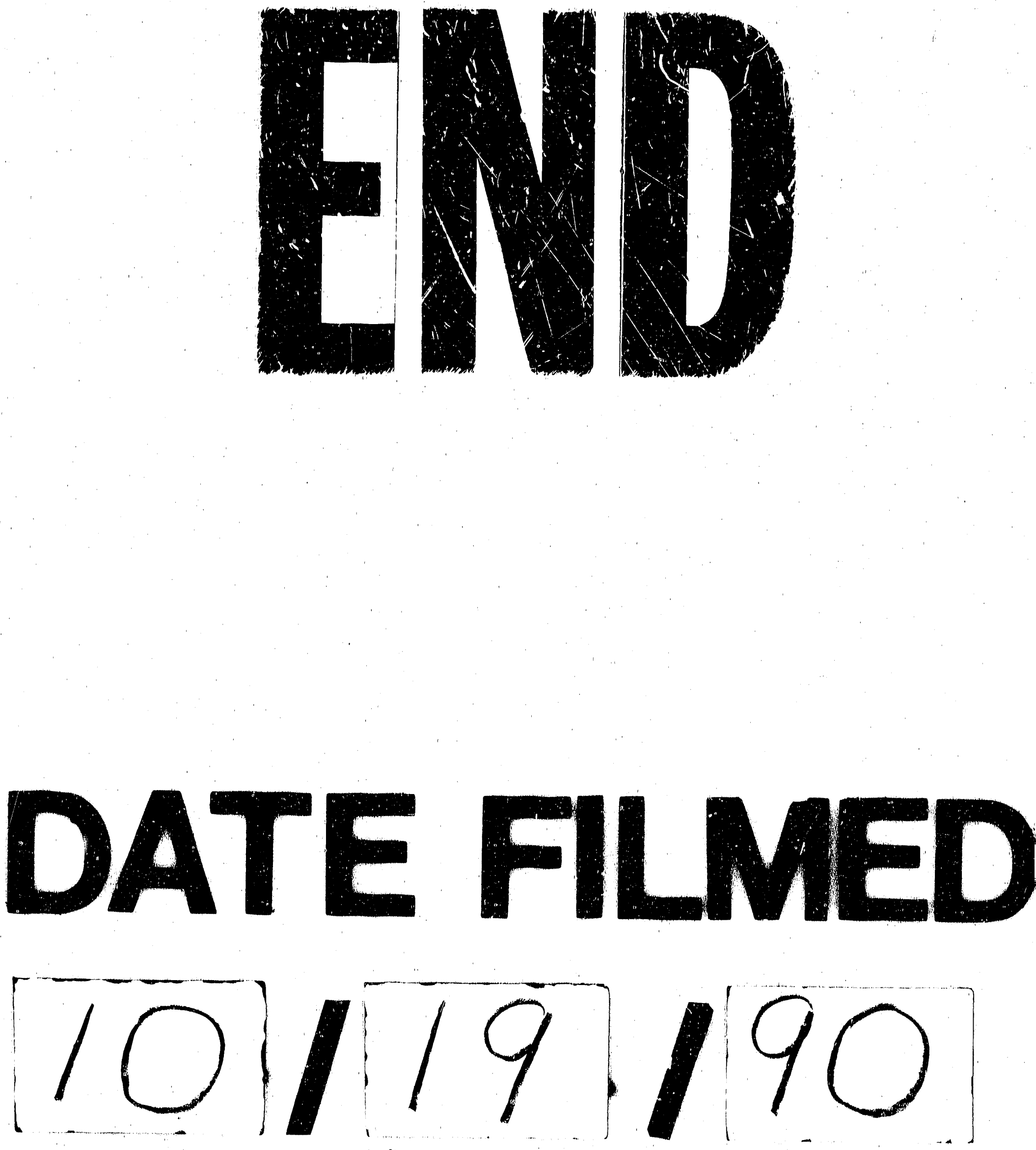

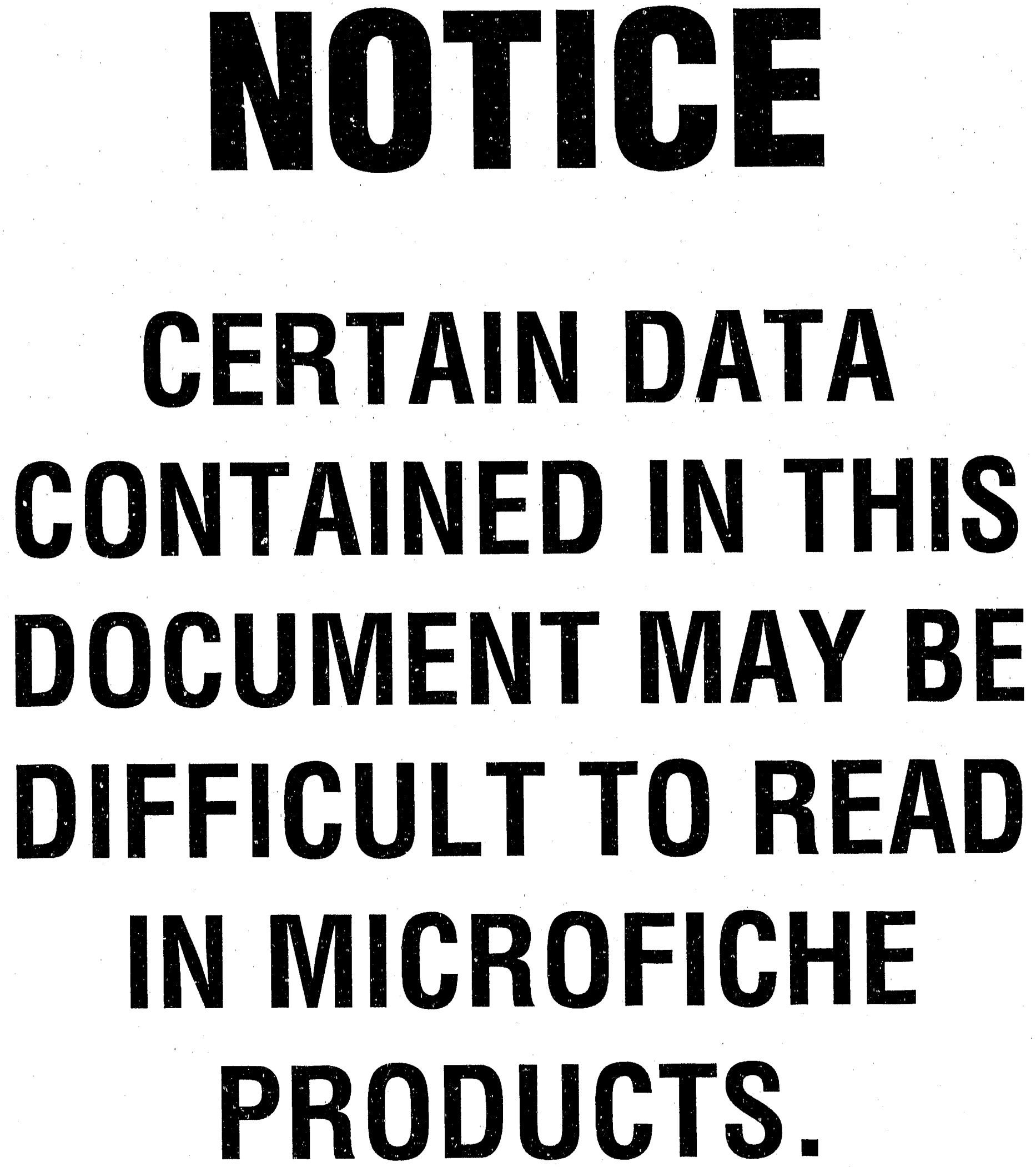


\section{LA-UR $90-3253$}

AUTHOR(S):

D. S. Sivia

W. A. Hamilton

G. S. Smith

DE9 1000215
SUBMITTED TO:

Proceedings:

"Maximum Entropy and Bayesian Methods: Laramie 1990"

T. Grandy \& L. Schick eds., Kluwer Academic Publishers

\section{DISCLAIMER}

This report was prepared as an account of work sponsored by an agency of the United States Government. Neither the United States Government nor any azency thereof, nor any of their employees, makes any warranty, express or implied, or assumes any legal liability or responsibility for the accuracy, completeness, or usefulness of any information, apparatus, product, or process disclosed, or represents that its use would not infringe privately owned rights. Reference herein to any specific commercial product, process. or serviç; by trade name, trademark, manufacturer, or otherwise does not necessarily constıtute or imply its endorsement, recommendation, or favoring by the United States Government or any agency thereof. The views and opinions of authors expressed herein do not necessarily state or reflect those of the United States Government or any agency thereof.

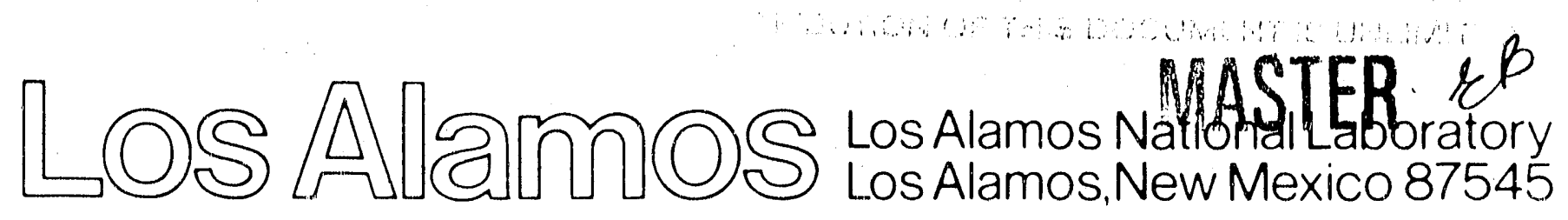




\title{
BAYESIAN SPECTRAL ANALYSIS OF REFLEC'TIVITY DATA
}

\author{
D. S. Sivia, W. A. Hamilton and G. S. Smith \\ Theoretical Division and Manuel Lujan Jr Neutron Scattering Center \\ Los Alamos National Laboratory \\ Los Alamos, New Mexico 87545, US.A
}

\begin{abstract}
The analysis of neutron and $x$-ray reflectivity data to obtain scattering-length density profiles is akin to the notorious phaseless Fourier problem, well-known in many fields such as crsytallography. Current methods of analysis culminate in the refinement of a few parameters of a functional model, and are often preceded by a long and laborious process of trial-and-error, A discussion of the use of maximum entropy for obtaining "free-form" solutions of the density profile, as an alternative to the trial-and-error phase when a functional model is not available, and the suggestion for a novel experimental procedure designed to alleviate the ambiguity problems inherent in traditional reflectivity measurements, are given in Sivia et al. (1990). In this paper we consider the Bayesian spectral analysis approach, which is appropriate for optimising the parameters of a simple (but adequate) type of model when the number of parameters is not known.
\end{abstract}

\section{Introduction}

A wide variety of interfaces of scientific and technological interest exhibit depth profile structure on length scales of tens to thousands of Angstrom. Examples include: model biological lipid membranes, analogous to the walls of living cells; semi-conductor multilayers of importance to the electronics industry; organic thin-film chemical sensors; conjugated polymers for electro-optic devices; and thin-film superconductors. This size range is an order of magnitude less than is accessible by conventional optical techniques. One method of probing interfacial structures on these length scales is to measure the interforence effects of the depth profile on the reflection of short wavelength radiation. This extension of visible range optical techniques to shorter wavelengths has been vigorously pursued over the last few years in $x$-ray reflectometry, and also by the increasingly popular complementary technique of neutron reflectometry.

In Section 2 we consider reflectivity from a data analysis point-of-view and in Section 3 we present the Bayesian spectral analysis approach to the problem. This is a generalised form of model-fitting where the solutions are restricted to a broad class of models rather than to a particular model. The choice of the type of modela encodes considerably more prior knowledge about the nature of the depth profile than does free-form approach (such as MaxEnt), while still allowing considerably more flexibility than conventional model refinement. We conclude with Section 4. 


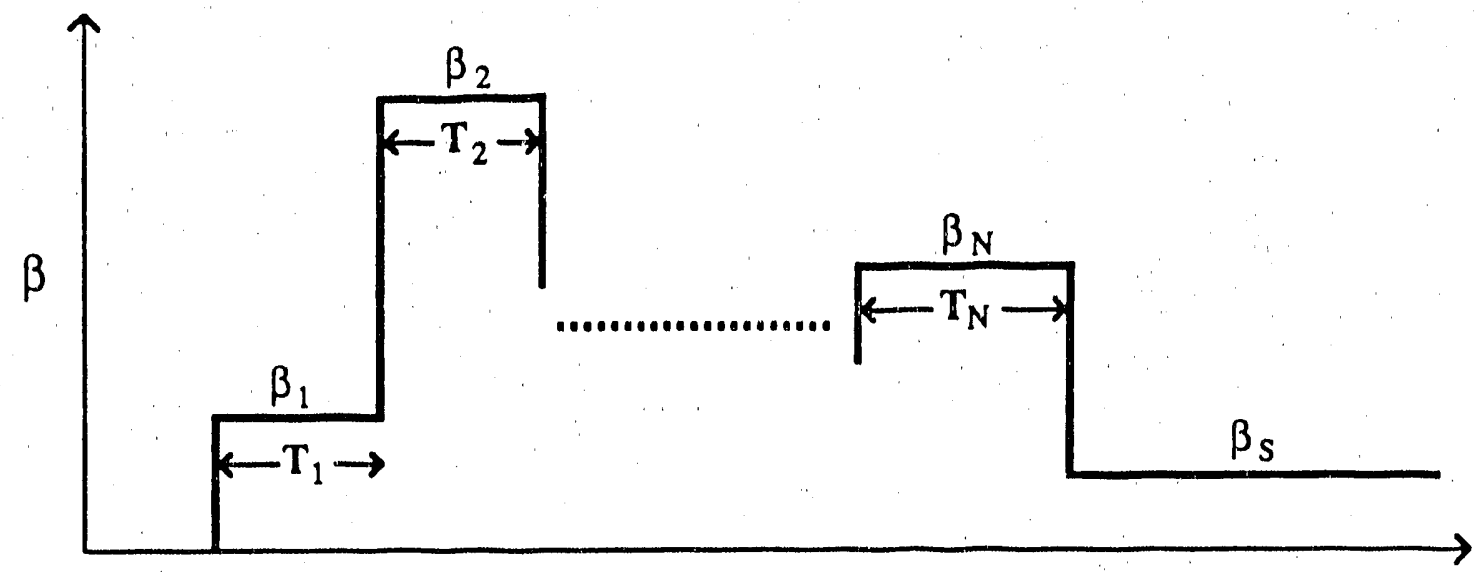

Figure 2. A simple, but (hopefully) adequate, representation of the scattering-length density profile based on the prior knowledge that layered structures have histogram-like depth profiles.

profile by constructing a hypothesis space defined by the "heights and widths" of a few layers as illustrated in Fig. 2. We could make a more elaborate model for the density profile by assigning a "roughness" parameter to each interface, and a "tilt angle" to each layer, but we ignore such complications for simplicity in this paper (the generalisation being straight-forward). This is also in keeping with the spirit of Bayesian spectral analysis where we are trying to estimate the parameters of a simple, but adequate, type of model when the number of parameters is not known. By using our additional prior knowledge to construct a much smaller hypothesis space (than that used in the free-form approach) we hope to obtain more accurate estimates of the depth profile, the reliability of which is also easier to quantify.

For this formulation of the problem, our best estimate of the density profile is obtained by maximising the posterior p.d.f.:

$$
\operatorname{prob}\left[T_{1}, T_{2}, \ldots, T_{N}, \beta_{1}, \beta_{2}, \ldots, \beta_{N}\left|R(Q), \beta_{S}\right|\right.
$$

where $\left\{T_{i}, \beta_{i}\right\}$ are the set of heights and widths of the $N$ layers describing the density profile, $\beta_{S}$ is the acattering-length density of the substrate (assumed known) and $R(Q)$ are the reflectivity data. If the number of layers was known, this would be a straight-forward problem of parameter eatimation (in principle). However, wo do not know NI So, we need to compute prob $\left[N \mid R(Q), \theta_{s}\right]$.

The mathematics presented below, to compute the posterior probability for the number of layers, is essentially the same as that found in Chapter 5 of Jeffreys (1939). It involves the use of Bayes' theorem and marginalisation, and requires a number of assumptions and approximations to be made in order to obtain an analytic solution. Although the assumptions and approximations are always open to question, they are not unusual in that they are always implicit when using the commonly-used least-squares analysis.

We begin by using Bayes' theorem: 

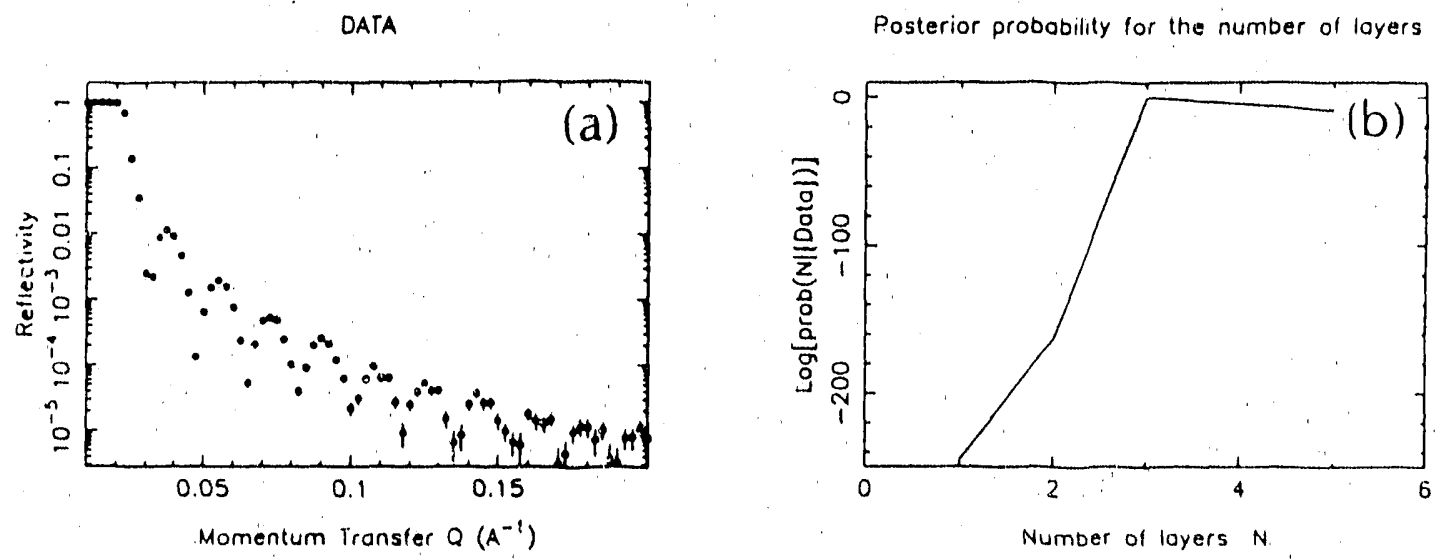

Posterior probability for the number of loyers

Best-fit density prolile assuming no. of layers $=3$
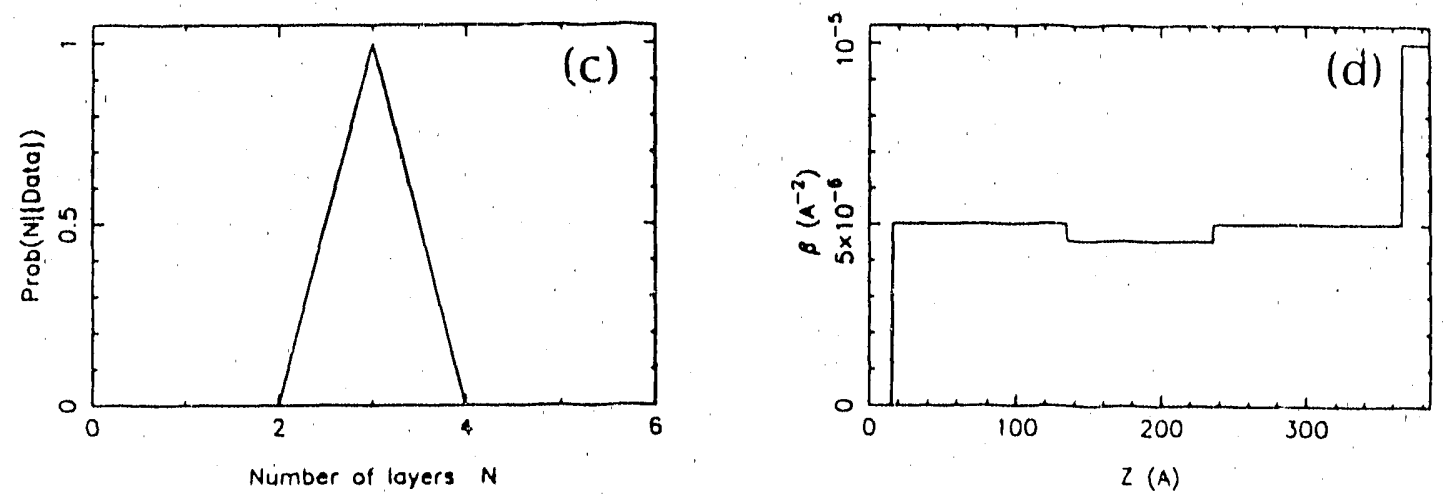

Figure 3. (a) Simulated reflectivity data. (b) \& (c) The posterior probability for the number of layers plotted on a logarithmic and linear scale respectively. (d) Best estimate of the density profile.

$$
\operatorname{prob}[N \mid R(Q)]=\frac{\text { Constant. } N !}{\left[\left(\beta_{\max }-\beta_{\min }\right) \cdot T_{\max }\right]^{N}} \times \frac{e^{-x_{\min }^{2} / 2}}{\sqrt{\operatorname{Det}\left(\nabla \nabla \chi^{3}\right)}}
$$

where $\operatorname{Det}\left(\nabla \nabla \chi^{2}\right)$ is the determinant of the Hessian matrix.

\section{AN EXAMPLE}

Fig. 3(a) shows simulated reflectivity data generated from a density profile consisting of a few layers; the simulated data suffer from typical instrumental resolution broadening and noise. Figs. 3 (b) and (c) show the posterior probability for the number of layers, computed according to the formula derived above. $T_{\max }$ was taken to be the actual width of the sample ( $350 \AA$ ) on the basis that, in general, it is very difficult to find the best-fit solutions if a good eatimate of the overall width is not available. $\beta_{\max }$ was set to $10^{-5}$ $A^{-2}$ in accordance with prior knowledge about the neutron scattering-lengths of atoms (the simulated density profile constructed to comply), $\beta_{\min }$ was set zero indicating prior 
the complex topology of the likelihood function for this non-linear problem means that it is very hard to find the best-fit solution, even when one knows the number of layers; a full parameter-space search entails cosmological tirne-scales even for models with only 4 or 5 layers! In practice we have found that our chances of success are greatly increased (above zero) when we have a good estimate of the total width of the sample.

\section{Conclusions}

Bayesian spectral analysis is a very powerful technique. It is a generalised form of modelfitting, which is appropriate for optimising the parameters of a simple (but adequate) type of model when the number of parameters is not known. In this paper we have the example of estimating the density profile for a layered sample, when the number of layers is unknown, but there are numerous other applications. Gull (1988), for example, addresses the problem of fitting a polynomial to a pertinent set of finite and noisy data, (again) when the order of the polynomial is not known.

This method of analysis encodes more prior knowledge about the object of interest than does a free-form approach, while still allowing a considerable amount of flexibility over traditional model refinement. Although we are often hesitant to commit ourselves to (even a clase of) simple functional models, the advantages of working in such a reduced hypotherio space are that we are able to obtain results which are more reliable and easier to quantify. It is in the nature of scientific inference that the best we can do is show that a theory (or choice of hypothesis space) is adequate (rather than "the truth"); if it is not adequate, probability theory will tell us so!

ACKNOWLEDGMENTS. We thank Roger Pynn for his encouragement and useful discussions. This work was supported by the Office of Basic Sciences of the U.S. Department of Energy.

\section{REFERENCES}

Felcher, G.P. (1988), 'Thin-Film Neutron Optical Devices', SPIE, 983.

Gull, S.F. (1988). 'Bayesian Inductive Inference and Maximum Entropy', in Maximum Entropy and Bayerian Methods in Science and Engineering (Vol. 1), eds. G.J. Erickson \& C.R. Smith, Kluwer Academic Publishers.

Jeffreys, H. (1939). Theory of Probability, Oxford University Press. Third edition: 1983.

Jaynes, E.T. (1986). 'Bayesian Methods - an Introductory Tutorial', in Maximum Entropy and Bayesian Methods in Applied Statistics, J.H. Justice ed., Cambridge University Press.

Lekner, J. (1987). Theory of the Reflection of Electromagnetic and Particle Waves, Martinez Nijof Publishers, Dordrecht, Holland.

Russell, T.P. (1990). 'X-ray and Neutron Reflectivity for the Investigation of Polymers', submitted to Materials Research Reports.

Sivia, D.S., Hamilton, W.A. and Smith, G.S. (1990). 'Analysis of Neutron Reflectivity Data: Maximum Entropy, Bayesian Spectral Analysis and Speckle Holograhy', Physica B, eds. G.P. Felcher and T.P. Russell (in press).

Thorburn, W.M. (1918). Mind, 27, 345-353. 

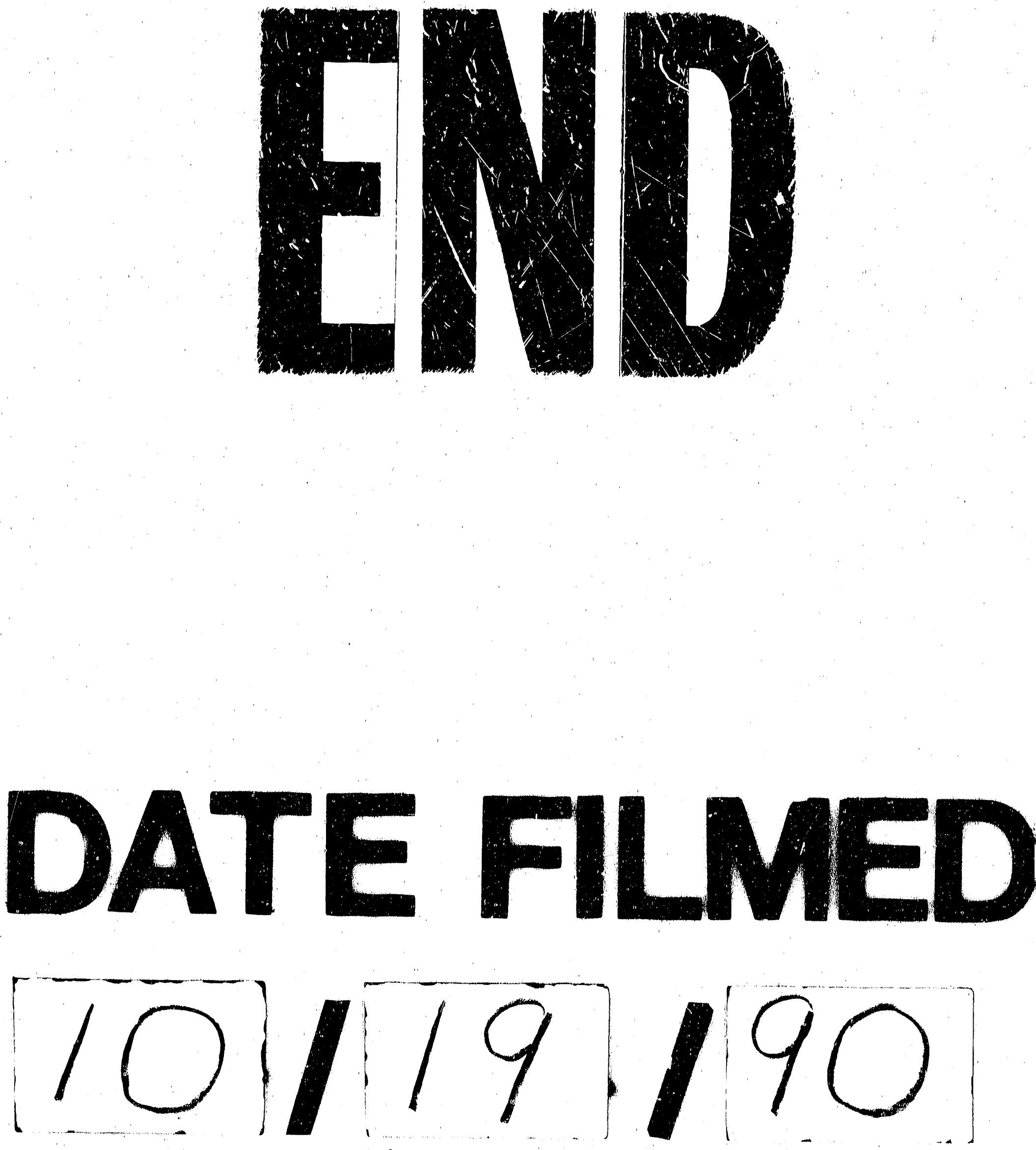
